\title{
Research on Garment Wrinkle Synthetic Method Based on Mass-Spring Model
}

\author{
Yuanwei Chen, Bing He, and Xuefu Yu
}

\begin{abstract}
The manifestation and details of garments are mainly represented by wrinkles. Through physical simulation of garment model, generated folding and wrinkles can be seen, The more mass points of garment mesh model are, the more realistic the effect are, but the longer the time will spend. On the contrary, the less mass points of garment mesh model are, the faster the calculation is, but the rougher the effect is. In order to give a balance to both simulation time and effect, this paper will show a enhanced wrinkle algorithm to conduct local-regional mesh refinement and simulation again providing a more detailed and realistic result for the simulation result of rough mesh.
\end{abstract}

Index Terms-Cloth simulation, garment, mass-spring, wrinkle.

\section{INTRODUCTION}

As a modern commodity, garments have the features of cold protection and covering body. it can also meet the requirements of people's life and work, and adapt various etiquettes and seasons. With the improvement of people's living standards, they are no longer satisfied with simple garments for body covering, but to pursue the garments with high quality, wearing comfort and good appearance. As to the garments themselves, their overall image is not only related to design level, fabrics quality, wearing comfort and surface quality, but also closely related to the appearance effect after garments wearing. Generated surface wrinkles by garments wearing is a direct factor impacting on wearing effect of garments and will greatly impact on the aesthetic feeling of appearance. Hence, it is very important and meaningful for research of generated wrinkles after garments wearing.

\section{RELATED WORK}

Wrinkles are an important appearance feature of garments and exist abundantly on the garments showing realness of garments. During garments wearing, wrinkles will be generated by bending deformation of flexible fabrics and human movement. Generated wrinkles by garments wearing are one of the important bases evaluating and comparing garments wearing effect. Therefore, it is necessary to conduct more detailed analysis on surface wrinkles of garments.

Current Research Status of Garment Wrinkle Simulation during Wrinkle Synthetic Process

Volino et al. [1] proposed a rapid geometric wrinkle

Manuscript received July 8, 2014; revised May 4, 2015.

The authors are with the State Key Lab of Virtual Reality Technology and Systems, Bei Hang University, China (e-mail: cywhezhl@163.com, Hebing@buaa.edu.cn). algorithm which could be added to any rough surface deformation model. The algorithm adjusts the range fixed at wrinkle height map in advance to achieve simulation with unchanging surface. Wrinkle sample plate is added on the initial deformation mesh and complex wrinkle deformation is synthesized by some wrinkle templates. Larboulette et al. [2] proposed a geometric limit method (length maintained) to form dynamic wrinkle which will appear or disappear according to the deformation of cloth. The previous research focuses on modeling or animation of garment and allows greater elastic deformation without considering surface extensibility in order to pursue visual effect. With reference to classical boundary triangularization concept of descriptive geometry, Tang et al. [3] proposed a new method to model extendable wrinkle surface by approximate extendable surface through bridge boundary triangularization method. Bridge boundary triangularization is achieved by minimum energy folding process of simulation cloth piece rotating from one end to the other end. Fu et al. [4] synthesized deformation simulation of wrinkle shape in the smooth surface area with fixed boundary and gave some basic parameter inputs such as wrinkle amplitude and extension.

Wang et al. [5] proposed adding rapid garment animation system of fine wrinkle details in the rough garment animation. The basis is that fine wrinkle can be approximately simulated by pre-calculated garment form data base of dynamic matching and overall animation form can be obtained by the rough simulation on the mesh with low resolution. It first builds a wrinkle data base of garment and then makes garment simulation based on high resolution in the influence area of joint based on skinned skeletal character model. It mainly simulates trousers and T-shirts closing to human body and almost continuous collision detection and little wrinkles are included for such kind of garments. They thought that wrinkles of underclothes are mainly generated by human motion and then proposed a wrinkle synthetic method based on the example, that is, wrinkle synthesis is driven by skeletal posture of human motion. The method allows that overall situation and dynamic part of cloth motion by the combination between cloth wrinkle of high accuracy and simulation of rough cloth. If the synthetic result is not consistent with referent simulation of high resolution, some special mid-scale characteristics and wrinkles shall be captured. In addition, synthetic system operates interactively and also supports unfeasible condition of offline simulation of high resolution. Wrinkle synthetic method uses synthetic model to obtain data base by cloth simulation pre-calculation during some posture movement. In principle, posture space is the exponential order of overall freedom degree, but fabric wrinkles are mainly affected by nearest node and each node can be operated independently. During synthesis, internal 
inserting of mesh is mainly for the influence of multi-node and final result can be obtained by interactive combination with rough simulation.

All the wrinkle simulation on the surface of garment can be achieved by the garment model with complete collision treatment and also can be achieved in the after-treatment through geometric wrinkle function. If it only has an analog for the wrinkle of garment from geometric angle, the number of triangles is remarkable. Even if it is in appropriate time intervals, such a big number of triangles will make the analog to garment not meet interactive demands in real time. If we can increase the number of triangles at the place where wrinkles will appear, calculation will be greatly reduced. Nadia Magnenat, Thalmann et al. [6] have made great progress in this aspect, but there is still a key question to solve, that is, how to estimate the area and direction for wrinkle appearing. If we want to achieve real analog for wrinkle of garment, friction force (especially friction force between garments and body) shall be considered and modeling for such friction force is hard. In this case, Thalmann et al. used a method based on geometry and texture (using rough mesh) to achieve analog for wrinkle and basic thought of this method lies in regional keeping of garment. The work is an extension based on the work of Gotoda [7], $\mathrm{Wu}$ [8] et al. and general process for the algorithm is as follows: First a wrinkle mode shall be defined (it is represented by texture and un-deformed triangle mesh in the initial state in a 3-D world). In the following calculation, texture mapping coordinates will not change. Four wrinkle coefficients of each triangle can be calculated by using wrinkle mode and initial mesh defined by the users and deformed mesh can be obtained by analog calculation to initial mesh. Form deformation between initial mesh and deformed mesh is calculated and modulation factor can be obtained by it and previously calculated wrinkle factor. Modulation mapping calculated by modulation factor can be used to modulate wrinkle mode. At last, final result can be obtained by synthesizing of modulated wrinkle mode and deformed mesh. Animation effect of garment wrinkle can be achieved by continuously implementing the above steps.

Decaudin et al. [9] proposed a complete geometric method for generating garment wrinkle. They used uneven shape generated by the garments surrounding columnar body of model as priori knowledge to synthesize process wrinkle of predefined type. The method requires that surface of columnar garments shall be added with predefined deformation type, but not random garment animation. People also proposed some other methods to synthesize process wrinkle effectively in the rough garment animation. Hadap[10] proposed a method based on texture, that is, wrinkle texture is synthesized by triangle deformation. $\mathrm{He}$ first calculated compressive strength drawing of garment surface deformation, and then used compressive strength drawing to balance local pre-rendered texture style so as to model wrinkle. Obtained texture can be used as protruding view of rapid wrinkle rendering. Kimmerle et al. [11] extended Hadap's method based on texture to texture synthetic mode taking compressive direction of triangle as original direction and deformation is achieved by displacement matching. The two methods can be used for animation rendering by multi-layer texture mixing at the price of not maintaining the area. Wrinkle direction is changing with time so wrinkle cannot be modeled by method of texture interpolation. And, frequency and width of wrinkle must be defined by the users in advance. Cutler et al. [12] defined garment wrinkle as curves inputting into cloth surface and these curves are taken as deformer synthesizing final shape. Shape and influence area of curves are established for specific series of frames artificially and corresponding wrinkle will appear or disappear appropriately when the middle frame is calculated. The method allows the painter to create expected wrinkle type which needs a lot of time and professional design capability anyway. Method of machine learning is adopted by Wang [5] and de Aguiar [13] to synthesize real-time wrinkle and example limiting application scope is needed to train data.

Damien et al. [14] also adopts process modeling method to synthesize real geometric wrinkle in the initial surface and proves the consistency of wrinkle changing with time. They describe the wrinkle with surface curve, but identification location and animation of curves are obtained by analyzing rough simulation result with time.

\section{WrinkLe AugMENTING AlgORITHM}

Wrinkle is not only a representation of uneven surface of object, but also a representation of internal performance of object. In the garment field, existence of wrinkles is universal and relationship among garment material, structure and performance can also be understood. Whether it is subjective factor or objective factor, complex dynamic wrinkles are shown on the sports garment and other clothes. It needs a time-consuming artificial design project or a simulation with high calculation price to synthesize such wrinkles in virtual environment

\section{A. Wrinkle Synthetic Process}

From current works of researchers, methods based on data base, modeling of geometry to wrinkle and physical analysis to wrinkle before refining are mainly for wrinkle synthesis. Main idea of the paper is to refine and augment result of rough simulation through analyzing region with larger deformation. Culter at el. [12] added program wrinkle model based on rough cloth simulation (wrinkles are artificially generated) and there are two obvious shortcomings for the method. First, artificial wrinkle mode is very time-consuming and needs professional skills. In addition, artificial setting can only be used for fixed frame, that is, real cloth wrinkles change continuously, deform with time and they can separate or blend dynamically.

Rohmer et al. [14] have improved the method, that is, an automatic real wrinkle generation method is used to replace artificial wrinkle model. The method can be used in any existing garment simulation, including spring simulation or skin covering model. Through research, we find that there is a close relation between wrinkle generation and approximate equal-distance relation among cloth extensibility, deformed garments and 2-D cloth pieces. Hence, tension of deformed cloth relative to static state can be taken as a reference for wrinkle displacement. To guarantee the time consistency of wrinkle animation, position and value of wrinkle change steadily. Actual wrinkle shape is synthesized by a new hidden 
surface deformation mechanism which supports complex wrinkle shapes including change of radius, width change of wrinkle and between wrinkles, and seamless transition of neighboring blending wrinkles.

Complex dynamic wrinkles are shown on sports garment and other clothes. It needs a time-consuming artificial design project or a simulation with high calculation price to synthesize such wrinkles in virtual environment which generally combines accurate parameter adjustment and needs professional animation skills. Rohmer gives a different wrinkle synthetic method, that is, rough simulation is made first and then real and fine wrinkles are synthesized during after treatment. Stretching tensor of rough simulation is taken as a reference for wrinkle displacement by us. To guarantee time consistency, a space-time method is adopted by displacement mechanism which not only allows smooth wrinkles, but also motion, separation and blending of wrinkles. Curved hidden deformer and automated method are adopted in the paper, and parameters controlled by the users can be used to simulate different cloth.

Wrinkles are the major manifestation and details of garment. Through physical simulation to garment mass-spring model, generated folding and wrinkles can be seen. The higher the model resolution is, the finer the simulation is. The lower the model resolution is, the rougher the simulation is. Model of high resolution will produce large simulation calculation quantity and it is very time-consuming Simulation calculation time is short for model of low resolution, but the details are not enough. Hence, to solve above problems, we make physical simulation to model of low resolution, analyze the simulation result, find maximum wrinkle point, spread externally and divide local wrinkle area subdivide the triangles of wrinkle area (that is, a large triangle is divided into 4 small triangles), fix boundary point of wrinkle area, make mass-spring simulation on internal vertex again and at last get a fine wrinkle effect. Simulation process is as follows:

1) Physical simulation for garment mass-spring model;

2) Analyze initial simulation result and find local maximum deformation point through calculating curvature;

3) Spread externally from the triangle where local maximum deformation point lies and divide local wrinkle area;

4) Subdivide in the local wrinkle area and increase meshes to improve local resolution of model;

5) Fix boundary point of local wrinkle area and make physical simulation on internal vertex;

Through twice physical simulation, a more detailed garment wrinkle simulation effect can be obtained.

\section{B. Method of Moreton and Sequin to Calculate Curvature}

When local maximum deformation point is selected, curvature is taken as the measurement criteria. First-order differential quantity of curved surface indicates tangent plane direction and normal vector, second-order differential quantity indicates concerned quantity such as curvature, and normal vector and curvature describe local geometric characteristics of triangular mesh model. But triangular mesh model is a representation form of discrete curve and there is no continuous normal vector and curvature for fabric piece, estimation is necessary for discrete curvature. Unified marks shall be introduced first.

$K$ represents Gaussian curvature, $H$ represents average curvature, $\bar{k}$ represents normal curvature, $k_{1}$ and $k_{2}$ represent principal curvature, and $n$ represents normal vector. In consideration of vertex $p_{i}$ of triangular mesh, $\left\{p_{j}\right\}_{j=0}^{m-1}$ represents a set of vertexes in its 1-neighborhood, $N(i)$ represents a set of indexes for vertexes in its1-neighborhood, $|N(i)|$ represents its vertex's degree, $\left\{T_{j}^{p_{j}}\right\}_{j=0}^{m-1}$ represents a set of triangular fabric pieces including point $p_{i}$, and $A\left(p_{i}\right)$ represents sum of triangular fabric pieces including point $p_{i}$..

Basic idea of the method is to use Euler's theorem of differential geometry to establish relations among normal curvature of curved surface, principal curvature of curved surface and principal direction. Take average value of all the normal vectors of triangular fabric pieces around point $p_{i}$, take it as normal vector of curved surface of triangular mesh at point $p_{i}$, and record it as $n$. Set $t_{j}$, which is unit projection of vector $p_{i} p_{j}$ on tangent plane of the curved surface of mesh, then normal curvature $\bar{k}_{j}$ of curved surface at point $p_{i}$ along direction $p_{i} p_{j}$ can be approximately taken as $p_{i}$ and $p_{j}$, and there is curvature of a circle for tangential $t_{j}$ at point $p_{i}$, that is:

$$
\overline{k_{j}}=2 \frac{\left(p_{j}-p_{i}\right) \cdot n}{\left(p_{j}-p_{i}\right) \cdot\left(p_{j}-p_{i}\right)}
$$

Let $b_{x}$ and $b_{y}$ be a group of basis on the tangent plane of curved surface of mesh, take $t_{j, x}$ and $t_{j, y}$ as coordinates of vector $t_{j}$ to the basis, and take $e_{x}$ and $e_{y}$ as coordinates of principal direction $e_{1}$ to the basis; from Euler's theorem, we can conclude that:

$$
\begin{aligned}
\overline{k_{j}} & =\left[\begin{array}{l}
t_{j, x} \\
t_{j, y}
\end{array}\right]^{T} \cdot \widetilde{K} \cdot\left[\begin{array}{l}
t_{j, x} \\
t_{j, y}
\end{array}\right] \\
\widetilde{K} & =\left[\begin{array}{ll}
e_{x} & e_{y} \\
-e_{y} & e_{x}
\end{array}\right] \cdot\left[\begin{array}{ll}
k_{1} & 0 \\
0 & k_{2}
\end{array}\right] \cdot\left[\begin{array}{ll}
e_{x} & e_{y} \\
-e_{y} & e_{x}
\end{array}\right]^{-1}
\end{aligned}
$$

Take $i, j=1,2, \ldots, m$ to obtain equation set $A x=b$, where

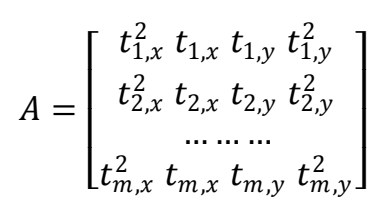

$$
x=\left[\begin{array}{c}
x_{0} \\
x_{1} \\
x_{2}
\end{array}\right]=\left[\begin{array}{c}
e_{x}^{2} k_{1}+e_{y}^{2} k_{2} \\
2 e_{x} e_{y}\left(k_{1}-k_{2}\right) \\
e_{x}^{2} k_{2}+e_{y}^{2} k_{1}
\end{array}\right], b\left[\begin{array}{c}
\widetilde{k_{1}} \\
\widetilde{k_{2}} \\
\cdots \cdots \cdots \\
\widetilde{k_{m}}
\end{array}\right]
$$

Given that

So

$$
x_{0}+x_{2}=k_{1}+k_{2}, 2 x_{0} x_{2}-\frac{x_{1}^{2}}{2}=k_{1} k_{2}
$$

$$
H=\frac{x_{0}+x_{2}}{2}, K=2 x_{0} x_{2}-\frac{x_{1}^{2}}{2}
$$




\section{Discrete Laplace-Beltrami Operator to Calculate Curvature}

Basic idea of the method is to introduce Laplace-Beltrami operator $\Delta=2 \mathrm{Hn}$ and average curvature manifold of curved surface, and $\Delta$ needs discretion by some means to obtain discrete value $\Delta_{M}^{k}\left(p_{i}\right)$ simply recorded as $\Delta_{M}^{(k)}$, and corresponding average discrete curvature is

$$
H=\Delta_{M}^{(k)} \cdot \frac{n}{2}
$$

Adopt Taubin method for discrete method of $\Delta$ in the experiment, as follows:

$$
\begin{gathered}
\Delta_{M}^{(1)}=\sum_{j \in N(i)} \omega_{i j}\left(p_{j}-p_{i}\right) \\
\sum_{j \in N(i)} \omega_{i j}=1
\end{gathered}
$$

Weighting factor meeting relation (4.10) can be determined by many means. Simply we can take

$$
\omega_{i j}=\frac{1}{|N(i)|}
$$

And also we can take

$$
\omega_{i j}=\frac{\varphi\left(p_{i}, p_{j}\right)}{\sum_{k \in N(i)} \varphi\left(p_{\mathrm{i}}, p_{k}\right)}
$$

Can be a sum of two triangular fabric pieces for side $p_{i} p_{j}$ and can also be a norm of length of side $p_{i} p_{j}$, such as

$$
\varphi\left(p_{i}, p_{j}\right)=\left\|p_{i}-p_{j}\right\|^{\alpha}
$$

We can take

$$
\omega_{i j}=1 /\left(2 \cot \propto_{i j}+2 \cot \beta_{i j}\right)
$$

Respectively for $\propto_{i j}$ and $\beta_{i j}$

$$
\propto_{i j}=\angle p_{i} p_{j-1} p_{j}, \beta_{i j}=\angle p_{i} p_{j+1} p_{j}
$$

Formula (4.13) is selected in the experiment.

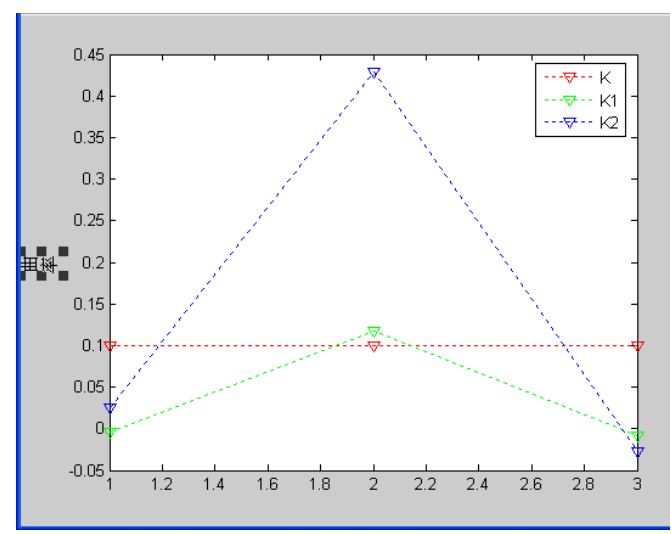

Fig. 1. Comparison of two curvature calculation methods.

D. Comparison of Two Curvature Calculation Methods

Above two calculation methods of curvature are applied to sphere and curvature calculation results are compared. Set the center of sphere in $(0,0,0)$ with radius of 10 . Surface parameter equation of sphere is:

$$
x^{2}+y^{2}+z^{2}=100
$$

If the curvature of any point on the sphere is $K=1 / r=0.1$, average curvature value of all points on the sphere is 0.1 . From above two curvature estimation methods, we calculate points $(0,0,10),(0,10,0)$ and $(10,0,0)$ to obtain vertex curvatures which are $K_{1}=\{-0.004576,0.117668,-0.009011\}, \overline{K_{1}}=0.034694$, $K_{2}=\{0.025486,0.428641,-0.026694\}$ and $\overline{K_{2}}=0.142478$ respectively. From average value, $\overline{K_{2}}$ is closer to K. From single value, $\overline{K_{1}}$ is closer to $\mathrm{K}$.

For operation time of the two curvature calculation methods, starttime and endtime can be used to indicate timing beginning and timing stopping. In calculation of 250 points, starttime and endtime of first method are $4 \mathrm{~s}$ and $4 \mathrm{~s}$ respectively, and starttime and end time of the second method are $5 \mathrm{~s}$ and $5 \mathrm{~s}$ respectively. In calculation of 500 points, starttime and endtime of first method are $12 \mathrm{~s}$ and $12 \mathrm{~s}$ respectively, and starttime and end time of second method are $17 \mathrm{~s}$ and $17 \mathrm{~s}$ respectively. We can see that time consumed by the two curvature calculation methods is short and there is almost no difference for calculation time.

\section{EXPERIMENTAL RESULTS}

Experimental Environment;

Operation System: Windows XP

CPU: Intel Quad-Core $2.40 \mathrm{GHz}$

Memory: 4.0GB

Programming Environment: VC++ and Direct X

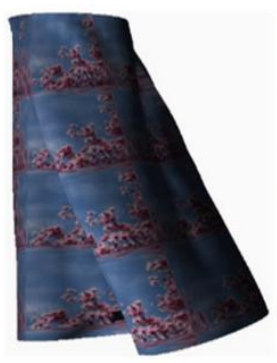

(a)

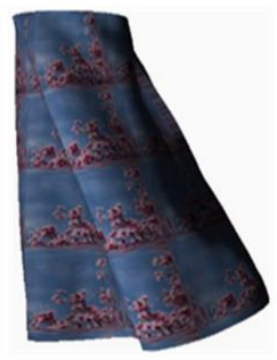

(b)
Fig. 2. Effect Comparison: (a) Rough simulation.(b) Refined simulation.

Simulation scene of the experiment is shown as Fig. 2 which imitates rough simulation of garment and simulation after wrinkle synthesis. Fabric piece number of textile is 512 and simulation step length is 0.01 . From rough simulation effect drawing (left), we can see that wrinkle is wide and big. From the effect drawing after wrinkle refining synthesis (right), wrinkle strip is more fine and real in detail.

\section{CONCLUSION}

The paper first concludes the forming reason of wrinkle and important meaning for garment performance, then concludes research achievements and methods of wrinkle synthesis in the garment simulation and at last gives wrinkle 
synthetic method above rough simulation which is suitable for the paper. That is, wrinkle area is divided through analyzing rough simulation result, mesh is refined by wrinkle area, boundary points are fixed and mass-spring physical simulation is made for internal points. Local wrinkles of garment will become more detailed after the synthesis.

\section{ACKNOWLEDGMENT}

This work was supported by grant No. 61272346 from NSFC (National Natural Science Foundation of China).

\section{REFERENCES}

[1] P. Volino and N. M. Thalmnn, "Fast geometrical wrinkles on animated surfaces," in Proc. the 7th International Conference in Central Europe on Computer Graphics and Visualization (WSCG), 1999, pp. 55-66.

[2] C. Larboulette and M.-P. Cani, "Real-time dynamic wrinkles," Computer Graphics International (CGI'04), vol. 6, pp. 522-52, 2004.

[3] K. Tang and C. C. L. Wang, "Modeling developable folds on a strip," Journal of Computing and Information Science in Engineering, vol. 5, pp. 35-47, 2005.

[4] F. Jing, A. Joneja, and K. Tang, "Modeling wrinkles on smooth surfaces for footwear design," Computer-Aided Design, vol. 37, pp. 815-823, 2005.

[5] H. Wang, H. Florian, R. Ravi et al., "Example-based wrinkle synthesis for clothing animation," in Proc. of ACM SIGGRAPH, 2010, vol. 29 no. 4 , pp. $1-8$

[6] P. Volino and N. M. Thalmann, "Animating wrinkles on clothes," in Proc. the 10th IEEE Visualization Conference(VIS99), 1999, pp. 175-523.

[7] T. L. Kunii and H. Gotoda, "Modeling and animation of garment wrinkle formation processes," in Proc. the Computer Animation 90, Tokyo: Springer, 1990, pp. 131-147

[8] Y. Wu, P. Kalra, L. Moccozet et al., "Simulation wrinkles and skin aging," The Visual Computer, vol. 15, no. 4, pp. 183-198, 1999.

[9] P. Decaudin, D. Julius, J. Wither, et al., "Virtual garments: A fully geometric approach for clothing design," Computer Graphics Forum (Eurographics'06 proc.), 2006, vol. 25, no. 3, pp. 625-634.

[10] S. Hadap, E. Bangerter, P. Volinoet al., "Animating Wrinkles on clothes," in Proc. the 10th IEEE Visualization 1999 Conference, 1999, pp. $175-182$

[11] S. Kimmerle, M. Wacker, C. Holzer, Multilayered Wrinkle Textures From Strain, VMV, pp. 225-232, 2004.
[12] L. Cutler, R. Gershbein, X. Wang et al., "An art-directed wrinkle system for CG character clothing," in Proc. the ACM/EG Symposium on Computer Animation, 2005, pp. 117-125

[13] E. de Aguiar, L. Sigal, A. Treuille et al., "Stable spaces for real-time clothing," ACM Transactions on Graphics (SIGGRAPH), vol. 29, no. 4, pp. $1-9,2010$.

[14] D. Rohmer, T. Popa, M.-P. Cani et al., "Animation wrinkling: Augmenting coarse cloth simulations with realistic-looking wrinkles," ACM SIGGRAPH Asia 2010 papers, vol. 29, no. 6, pp. 1-8, 2010.

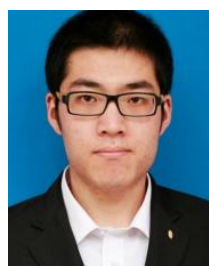

Yuanwei Chen was born in 1990, Chengde, Hebei province. From 2009 to 2013, he studied in Tianjin Polytechnic University majoring in soft engineering. In 2013, he passed the exam of computer science in BUAA and will graduate in 2016. During the period of master, his main research is the cloth simulation. $\mathrm{Mr}$ Chen has 1 item 1 authorized patents.

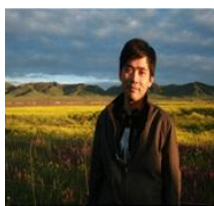

Bing He was born in 1971, he is an associate professor In 2001, he obtained doctoral degree from Department of Electronic Engineering of BUAA. Since 2001, he has been engaged in the teaching and scientific research in BUAA institute of computer virtual reality technology and system state laboratory. His main research areas include virtual reality, video and image processing, information visualization, embedded system, information fusion and intelligent traffic, etc.

Prof. He has published in core journals and academic conference at home and abroad more than 30 papers. Successively presided over many national 863 plan project, pre-research project and horizontal topic, and won the provincial science and technology progress award, 1 item 1 authorized patents.

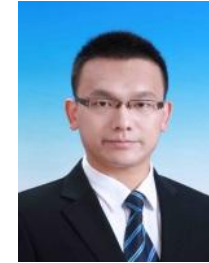

Xuefu Yu was born in 1988, Tianjin, From 2007 to 2011, he studied in Tianjin University majoring in computer science. In 2014, he obtained academic master degree of computer science of BUAA. During the period of master, his main research is the cloth simulation. Mr. Yu has 1 item 1 authorized patents. 